\title{
Seasonal density dependence, timing of mortality, and sustainable harvesting
}

\author{
Hanna Kokko *, Jan Lindström \\ Department of Zoology, University of Cambridge, Downing Street, Cambridge, CB2 3EJ, UK
}

Received 20 January 1998; accepted 15 April 1998

\begin{abstract}
Birth-pulse populations are often characterized with discrete-time models, that use a single function to relate the post-breeding population size to the post-breeding size of the previous year. Recently, models of seasonal density dependence have been constructed that emphasize interactions during shorter time periods also. Here, we study two very simple forms of density-dependent mortality, that lead to Ricker and Beverton-Holt type population dynamics when viewed over the whole year. We explore the consequences of harvest timing to equilibrium population sizes under such density dependence. Whether or not individual mortality compensates for the harvested quota, the timing of harvesting has a strong impact on the sustainability of a harvesting quota. Further, we show that careless discretization of a continuous mortality scheme may seriously underestimate the reduction in population size caused by hunting and overestimate the sustainable yield. We also introduce the concept of the demographic value of an individual, which reflects the expected contribution to population size over time in the presence of density dependence. Finally, we discuss the possibility of calculating demographic values as means of optimizing harvest strategies. Here, a Pareto optimal harvest strategy will minimize the loss of demographic value from the population for a given yield. (C) 1998 Elsevier Science B.V. All rights reserved.
\end{abstract}

Keywords: Beverton-Holt and Ricker models; Compensatory mortality; Doomed surplus; Hunting season timing; Maximum sustainable yield; Population harvesting

\section{Introduction}

Seasonally reproducing species that produce offspring during a short annual period have birth-

* Corresponding author. Tel.: + 441223 336627; fax: + 44 1223 336676; e-mail: h.kokko@zoo.cam.ac.uk pulse dynamics usually described with discrete time population models (Caswell, 1989). When the model includes effects of population density on demographic parameters, the customary approach is to use a single function over the year relating, for instance, population growth to population density. However, the effect of population 
density on mortality in seasonally reproducing animals typically varies during a year, being often at its highest on newly-born offspring (Ekman, 1984; Clutton-Brock et al., 1985; Skogland, 1985; Hudson, 1992; Clutton-Brock et al., 1997). Also, it has been shown that seasonality in density dependence may have profound consequences for population dynamics (Kot and Schaffer, 1984; Rodriguez, 1988; Åström et al., 1996) and, consequently, on optimal harvesting strategies (Allen et al., 1991).

Here, we show the strong effect that seasonality has on population persistence, defined as the ability to tolerate additional mortality. Harvesting theory takes advantage of this tolerance, exploiting the capacity of populations to give birth to more individuals than can survive in their environment. Here, the important effect of the structure of density dependence is whether hunting mortality is simply additive to natural mortality or partly or fully compensated. In seasonal, terrestrial environments, such survival refers mainly to over-wintering populations with limited resources. The fraction of the population likely to die during winter even without any hunting was named the 'doomed surplus' by Errington (1934). By his argument, hunting would not decrease the subsequent breeding population if the number of individuals killed does not exceed the size of the 'doomed surplus'.

Errington's (1934) view, however, represents only one extreme of the possible outcomes of extra mortality. The actual outcome here depends on the type of critical factor causing density-dependent mortality during the period of kills. If, for instance, population density affects the efficiency of an individual to gather food reserves for wintering, it may be that removing individuals by hunting after a certain period does not improve the survival probability of the remaining individuals. An example of the other extreme of a corresponding scenario is that winter food itself is a critical factor for surviving over winter. That being the case, removal of an individual anytime before spring may immediately help the rest of the population to survive and thus create the possibility for a 'doomed surplus' to exist. Studies aimed at determining whether hunting mortality is to- tally compensated (or even overcompensated), or partly or wholly additional in natural populations, have not given an unequivocal result, although totally compensatory mortality is probably rare (Anderson and Burnham, 1976; Roseberry, 1979; Conroy and Eberhardt, 1983; Burnham and Anderson, 1984; Burnham et al., 1984; Payne, 1984; Robertson and Rosenberg, 1988; Barker et al., 1991; Ellison, 1991; Small et al., 1991; Dusek et al., 1992; Rexstad, 1992; Smith and Reynolds, 1992, 1994; Sedinger and Rexstad, 1994; Hellgren et al., 1995).

Sustainable harvesting schemes can be built for both additive and compensatory hunting mortality but, if the aim is to take advantage of compensatory survival, timing of density-dependent phenomena must be specified, as well as the effect of the removal of competing individuals. Existing harvest strategies surely take this into account: The usual practice in harvesting terrestrial birthpulse populations is to permit harvesting only after the breeding season in autumn. Common sense states that the opposite alternative of harvesting before reproduction means deducting from the capital, hence the stable population size and sustainable yield would be much reduced by adopting such a strategy. However, very little theoretical work has been devoted to study the foundations of this argument (Doubleday, 1975; Getz, 1980).

The aim of this study is to show the importance of timing differences. Also, it shows the potential for serious misinterpretation if a continuous population model is discretisized carelessly and, specifically, if discrete-time harvesting models are used in a population where harvest mortality is continuous (even if constrained to a small timespan annually).

\section{Models of density dependence}

Throughout the following models, we consider a 'birth-pulse' population (Caswell, 1989) with breeding occurring at time steps $0,1,2, \ldots$, maturation at the age of 1 year, and with density-dependent mortality between the breeding seasons. The breeding function 


$$
B: x \rightarrow \lambda(x) x
$$

relates the post-breeding population size to the pre-breeding population size, with a per capita multiplication rate of $\lambda(x)$.

Unless otherwise stated, equilibrium population sizes are measured just after the birth-pulse. We consider the time interval $(0,1)$ only, starting with a post-breeding population size and ending with the birth-pulse at time step 1. At equilibrium, these two sizes must be equal, and subsequent time intervals will only repeat the pattern observed within the time interval $(0,1)$.

Let the initial post-breeding population size be $x(0)$. During the time interval $(0,1)$, individuals die with death rate $\mu(t)$,

$\frac{\mathrm{d} x}{\mathrm{~d} t}=-\mu(t) x(t)$

where density dependence can be introduced by letting $\mu$ depend on the population size, $x$. The mortality function $M$ relates the pre-breeding population size at time 1 to the post-breeding population size of time 0 :

$M: x(0) \rightarrow x(0)+\int_{t=0}^{1}-\mu(t) x(t) \mathrm{d} t$

The mapping $f(x)$ of population size from 1 year to another now becomes

$x(1)=f(x(0))=B(M(x(0)))$

We start with the case with no density dependence in the breeding season, which is obtained by setting $\lambda(x)=\lambda_{0}$ for all $x$. We consider two scenarios for the death rate, $\mu(t)$ : (1) $\mu(t)$ depends on the current population size, such that every death in the population is compensated by higher survival of the individuals left: $\mu(t)=f\left(\mu_{0}, x(t)\right)$; (2) $\mu(t)$ is constant for a single season and depends on the state of the environment, determined by population size, at the start of the season only: $\mu(t)=f\left(\mu_{0}, x(0)\right) \forall t \in(0,1)$. Variations in $\mu(t)$ irrespective of $x$ are considered later.

Specifically, for (1), choosing $\mu(t)=\mu_{0} \cdot x(t)$ and a constant multiplication rate $\lambda(x)=\lambda_{0}$ gives the Beverton-Holt equation (Beverton and Holt, 1957), $\frac{\mathrm{d} x}{\mathrm{~d} t}=-\mu_{0} \cdot x(t)^{2}(0 \leq t<1)$,

$\lim _{\varepsilon \downarrow 0} \frac{x(1)}{x(1-\varepsilon)}=\lambda_{0} \Rightarrow x(1)=\frac{\lambda_{0} x(0)}{1+\mu_{0} x(0)}$.

Likewise, the choices $\mu(t)=\mu_{0} \cdot x(0)$ and $\lambda(x)=\lambda_{0}$ give the Ricker equation (Ricker, 1954):

$\frac{\mathrm{d} x}{\mathrm{~d} t}=-\mu_{0} x(0) x(t)(0 \leq t<1)$,

$\lim _{\varepsilon \downarrow 0} \frac{x(1)}{x(1-\varepsilon)}=\lambda_{0} \Rightarrow x(1)=\lambda_{0} x(0) \mathrm{e}^{-\mu_{0} x(0)}$

Without harvesting, the stable equilibrium of the population size is $x^{*}=\left(\lambda_{0}-1\right) / \mu_{0}$ for the Beverton-Holt model, and $x^{*}=\ln \left(\lambda_{0}\right) / \mu_{0}$ for the Ricker model (Getz and Haight, 1989). These are sizes measured immediately after the birth-pulse; the equilibrium sizes before the birth pulse are obtained by dividing by $\lambda_{0}$.

\section{The effect of harvest timing}

The sustainable use of a population basically relies on its density-dependent response to a decreased number of competitors (Getz and Haight, 1989). If one is not interested in the timing of harvesting, one might write in the Beverton-Holt model

$x(1)=\frac{\lambda_{0} x(0)}{1+\mu_{0} x(0)}-H$

or in the Ricker model

$x(1)=\lambda_{0} x(0) \mathrm{e}^{-\mu_{0} x(0)}-H$

and solve for $x(0)=x(1)$ to get the equilibrium population size for a harvest quota. In practice, however, all harvesting cannot be focused to an infinitesimally short time period, and it is not immediately clear how much this will affect the stable population size. We therefore solve for the following equilibrium:

Let the total harvesting quota equal $H$, uniformly divided within the time interval $\left(T_{1}, T_{2}\right)$ $\left(0 \leq T_{1}<T_{2} \leq 1\right)$, with $h=H /\left(T_{2}-T_{1}\right)$ individuals removed per unit time: 


\section{Beverton-Holt}
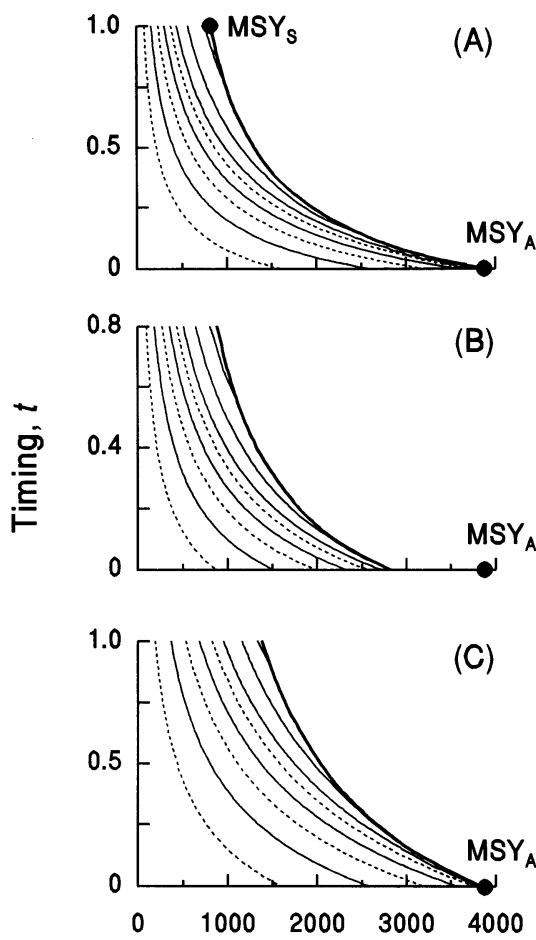

Ricker

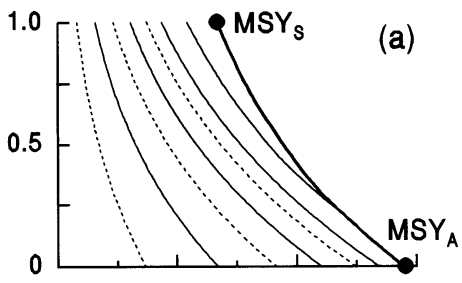

(b)
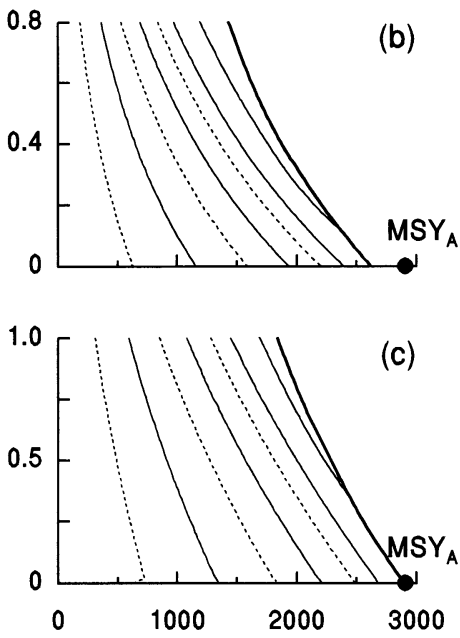

(c)

Total bag, $H$

Fig. 1. Equilibrium population sizes (measured immediately after the birth-pulse) for the harvested Beverton-Holt and Ricker models. Parameter values are $\lambda_{0}=5$ in both cases, and $\mu_{0}$ is adjusted to give equilibrium population size 10000 in a non-harvested population in both cases $\left(\mu_{0}=0.0004\right.$ for the Beverton-Holt model and 0.00016 for the Ricker model). Total bag is given on the $x$-axis, the timing choice on the $y$-axis, and the contours give equilibrium population sizes as follows: solid lines from left to right give the contours for $90,80,70,60$ and $50 \%$ of the population left, and the extinction contour $(0 \%)$. Dotted lines from left to right give additionally 95,85 and $75 \%$ contours. Point $(0,0)$ always has population size $10000(100 \%)$. In the region to the right of the extinction contour, harvesting is always unsustainable. Timing choices: (A-a), all harvesting occurs in a single pulse at a specific time $t$ between 0 and 1 . (B-b), harvesting

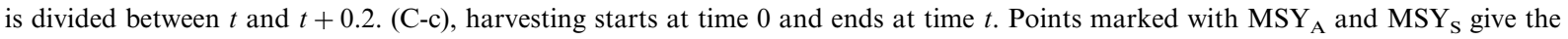
maximum sustainable yield if all harvesting occurs immediately after (autumn MSY) or prior (spring MSY) of the birth-pulse.

$\frac{\mathrm{d} x}{\mathrm{~d} t}= \begin{cases}-\mu(t) x(t), & 0 \leq t<T_{1} \\ -\mu(t) x(t)-h, & T_{1} \leq t<T_{2} \\ -\mu(t) x(t), & T_{2} \leq t<1\end{cases}$

The solution of Eq. (7) gives the (fairly complicated) equation that the equilibrium population size has to satisfy, and it is fully presented in Appendix A for both Beverton-Holt and Ricker formulations. The equilibrium will dramatically depend on harvest timing (Fig. 1). Too late harvesting may overexploit a population to extinction even if the same quota would only slightly reduce the original population size, if it had been removed early in the season: e.g. in Fig. 1A, a bag of 1500 individuals harvested immediately after the birth-pulse will reduce the equilibrium population size by a mere $5 \%$, but the reduction is $20 \%$ if one fourth of the season has passed before harvesting occurs, and the population is driven to extinction if the removal happens after more than a third of the season. This is especially remarkable, given that the bag of 1500 is much below the maximum sustainable yield $\left(\mathrm{MSY}_{\mathrm{A}}\right)$ of almost 4000 individuals (Fig. 1A). Note also that even in this deterministic setting, the MSY as an absolute 
maximum to the tolerable bag is sustainable only if the harvesting season is extremely short. There is no way of having a sustainable bag approach the MSY if the hunting season is of considerable length (Fig. 1B-b). The sensitivity for harvest timing is similar in short (Fig. 1A-a) and intermediately long (Fig. 1B-b) harvest seasons as well as in a setting where the open season begins at the end of the breeding-pulse but varies in length (Fig. 1C-c). Also, the timing matters both in cases where the survival of the remaining individual increases such that it compensates for the loss of others (the Beverton-Holt model, Fig. 1A-C) and in cases where it does not (the Ricker model, Fig. 1a-c), although the response to timing is less pronounced in the latter case.

\subsection{Finding the extremes: spring versus autumn mortality}

We now show the dangers of careless discretization, by imagining that we only wish to compare harvesting schemes that occur just prior or just after the birth-pulse, and to seek the respective maxima of sustainable harvesting. This corresponds to finding only the $\mathrm{MSY}_{\mathrm{A}}$ and $\mathrm{MSY}_{\mathrm{S}}$ points in Fig. 1A-a, which should at least give some impression of the sensitivity of the systems to harvest timing. The total harvest quota, $H$, is now divided into spring harvest, $H_{\mathrm{s}}$, and autumn harvest, $H_{\mathrm{a}}$. Using Eqs. (1)-(3), the discrete dynamics becomes

$x(1)=B\left(M\left(x(0)-H_{\mathrm{a}}\right)-H_{\mathrm{s}}\right)$

The relationship between $H_{\mathrm{a}}, H_{\mathrm{s}}$ and the stable population size $x^{*}$ is derived by solving for the stable equilibrium population size in Eq. (8): $x *$ is the solution of $x(0)=x(1)$. There are two solutions, of which the larger one is stable. At the point where the two solutions coincide, the MSY is reached (Fig. 2).

For the Beverton-Holt model, we have $B(x)=$ $\lambda_{0} x$ and $M(x)=x /\left(1+\mu_{0} x\right)$, and the requirement of the equality of the two solutions leads to the expression

$H_{\mathrm{a}}^{*}+\lambda_{0} H_{\mathrm{s}}^{*}=\frac{\lambda_{0}-2 \sqrt{\lambda_{0}}+1}{\mu_{0}}$
In the example of Fig. 1A, this means that if spring harvesting $H_{\mathrm{s}}=0$, the maximum autumn harvest is $\mathrm{MSY}_{\mathrm{A}}=3820$, whereas this figure has to be divided by $\lambda_{0}$ to get the maximum spring harvest, 764. Indeed, from Eq. (9) it follows that for every individual removed in spring, the autumn bag has to be removed by $\lambda_{0}$, to keep the yield sustainable. This is not surprising, given that the breeding season brings about a multiplication of the population by the factor $\lambda_{0}$.

The Ricker formulation, $B(x)=\lambda_{0} x$ and $M(x)=x \mathrm{e}^{-\mu_{0} x}$, gives similar results at first sight. Solving for the equilibrium population size in Eq. (8) gives an implicit solution

$x^{*}=H_{\mathrm{a}}+\frac{C}{\mu_{0}}$

where $C$ fulfils $C\left(\lambda_{0} \mathrm{e}^{-C}-1\right)=\mu_{0}\left(H_{a}+\lambda_{0} H_{s}\right)$. The value of $C$ cannot be solved analytically, but

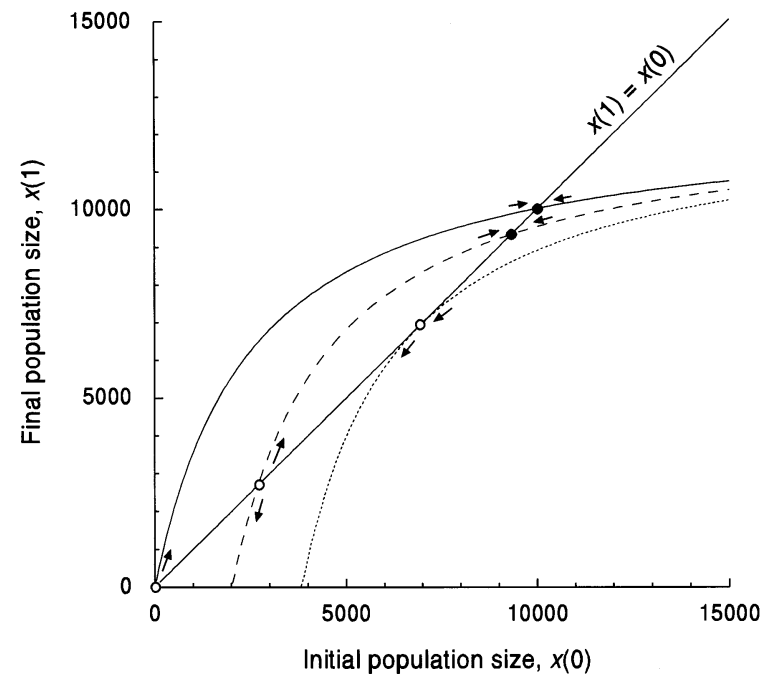

Fig. 2. Derivation of the stable equilibria and the MSY, exemplified for the Beverton-Holt model with autumn hunting only. Parameters are as in Fig. 1. Solid line: Population growth without hunting, $x(1)=\lambda_{0} /\left(1+\mu_{0} x(0)\right)$, gives the equilibrium population size of 10000 individuals (filled dot) and the trivial unstable equilibrium 0 (open dot). Dashed line: Population growth with an autumn bag of 2000 individuals, $x(1)=\lambda_{0} /\left(1+\mu_{0}(x(0)-2000)\right)$, reduces the stable population size but increases the value of the unstable equilibrium. Dotted line: The $\mathrm{MSY}_{\mathrm{A}}$ hunting, giving the map $x(1)=\lambda_{0} /(1+$ $\left.\mu_{0}(x(0)-3820)\right)$, decreases population growth enough to make the two solutions (the stable and the unstable equilibrium) converge to a single, unstable equilibrium. 
it can be shown that the curve $C\left(\lambda_{0} \mathrm{e}^{-C}-1\right)$ has a unique and positive maximum where the two solutions coincide. Denoting its value by $V$, one readily sees that the maximum harvest quotas $H_{\mathrm{a}}^{*}$ and $H_{\mathrm{s}}^{*}$ that lead to the same equilibrium population size are related by $H_{\mathrm{a}}^{*}+\lambda_{0} H_{\mathrm{s}}^{*}=V / \mu_{0}$. Again, for each individual removed in spring, autumn harvesting $H_{\mathrm{a}}$ has to be decreased by $\lambda_{0}$ individuals if sustainability is to be maintained. Note that while the post-breeding equilibrium population size $x^{*}$ depends on $H_{\mathrm{a}}$ as well as on the sum $H_{\mathrm{a}}+\lambda_{0} H_{\mathrm{s}}$, the autumn population size after harvesting, $x^{*}-H_{\mathrm{a}}$, depends only on the latter and equals $V / \mu_{0}$.

So far, both the Beverton-Holt and the Ricker model have been shown to satisfy the idea that one individual removed in spring corresponds to $\lambda_{0}$ individuals removed in autumn, a result not surprising given the per capita rate of multiplication, $\lambda_{0}$, that occurs in the birth pulse. However, the continuous-time model predicts that only the Beverton-Holt model has a five-fold $\mathrm{MSY}_{\mathrm{A}}$ compared to $\mathrm{MSY}_{\mathrm{S}}$ (Fig. 1A) — the Ricker model shows only about a two-fold difference (Fig. 1a).

One needs to be certain of the implicit assumptions made by using Eq. (8) to form this conclusion. Specifically, Eq. (8) is formulated such that autumn hunting occurs fast enough to cancel the effects of high post-breeding population size on over-winter mortality: it is assumed that population size after autumn hunting, $x(0)-H_{\mathrm{a}}$, and not the post-breeding population size, $x(0)$, determines the state of the environment that generates the density dependence (Eq. (8)). But if conditions for density dependence are effectively determined by the breeding population, autumn hunting occurs too late to prevent environmental deterioration. If this is true, the usage of Eq. (8) to describe the situation is invalid. In the mortality function $M$, density dependence should act according to the post-breeding density $x(0)$, while the number of individuals actually entering the non-breeding season should be $x(0)-H_{\mathrm{a}}$. Eq. (6b) has to be replaced by the explicit expression

$$
x(1)=\lambda\left(\left(x(0)-H_{\mathrm{a}}\right) \mathrm{e}^{-\mu_{0} x(0)}-H_{\mathrm{s}}\right)
$$

This equation gives the equilibrium population size $x^{*}=D / \mu, D$ being the solution of $D\left(\lambda_{0} \mathrm{e}^{-D}\right.$ $-1)=\mu_{0} \lambda_{0}\left(H_{\mathrm{s}}+\mathrm{e}^{-D} H_{\mathrm{a}}\right)$. This form predicts correctly the MSY points in Fig. 1. Harvesting in spring has in this case much less severe consequences since it helps to reduce the post-breeding population size, which has partly negative effects on population growth through density dependence.

\subsection{Doomed surplus and the demographic value of an individual}

At first sight, it may seem strange that a population tolerates more extra mortality if the harvest quota is removed as early in the season as possible, even in the case where the death of culled individuals does not affect the remaining individuals' death rate in any way. However, the case becomes clear when one considers the expected survival probability of an individual alive at time $t<1$ up to the beginning of the breeding season at time 1. With no compensation (constant death rate $\mu)$, this probability equals $\mathrm{e}^{-\mu(1-t)}$, where $1-t$ is the length of the remaining season to over-winter. Culling that individual reduces its survival probability to zero, and thus removes an expectation of $\mathrm{e}^{-\mu(1-t)}$ individuals from the population. The expectation increases as the season proceeds: survived individuals gain demographic value during the mortality season. The gain is strengthened by compensation, but it exists even without it. Here, we note that although the Ricker model represents overcompensation when interpreted on a yearly basis (Reed, 1980), the withinseason mortality leading to Ricker type dynamics shows no compensation on the basis of individual mortality, and the only deviation from additive mortality results from the above phenomenon of time-dependent survival expectations.

We illustrate this effect with the concept of demographic value of an individual. It is well known that a successful harvesting strategy should spare individuals with high reproductive value (MacArthur, 1960; Caughley, 1977; Yodzis and Kolenosky, 1986; Goodyear, 1996; Kokko et al., 1997). However, even if individuals do not differ in any respect, the demographic value (measured as the reduction in the subsequent population size) of removing the first $n$ individuals does 
not equal that of removing the next $n$. Also, the timing of removal matters, as shown above.

To formalize the concept of demographic value, consider a map $f$ from population size at time $t_{1}$ to size at $t_{2}$. We define the demographic value $v_{D}$ of an individual removed from the population at time $t_{1}$ to equal the drop in population size, resulting from that removal, measured at a future reference time point $t_{2}$. If all individuals are equal, the value of $v_{D}$ depends on population size $x$ only:

$v_{D}(x)=f(x)-f(x-1)$

where $f$ defines the mapping $f: x\left(t_{1}\right) \rightarrow x\left(t_{2}\right)$. Thus, the greater the slope of $f(x)$, the higher is the demographic value of one individual removed (Fig. 3A). A 'doomed surplus' is established, if there is a region in $f$ where $v_{D}(x) \leq 0$. The width of the region gives the number of individuals belonging to this surplus (Fig. 3B).

A sustainable harvesting strategy, however, does not need to be confined to using the 'doomed surplus' only. Harvesting mortality does not need to be completely or even partially compensated to make harvesting sustainable (Robertson and Rosenberg, 1988). Here, in terms of the demographic value, a specific type of surplus is created by the harvest itself in the following way. If the map $f$ is a complete description of the dynamics of the population from one breeding season to another, it can be used to determine equilibrium population sizes and responses to harvesting (Fig. 3C). Harvesting reduces population size each year

Fig. 3. Illustrations of the demographic value concept. (A) Depending on the slope of the mapping $f: x\left(t_{1}\right) \rightarrow x\left(t_{2}\right)$, the effect of removing one individual from the population is magnified (at $x_{1}$ ) or diminished (at $x_{2}$ ) by density dependence. The demographic value can be even negative, if the removal of an individual increases the subsequent population size (at $x_{3}$ ). (B) A 'doomed surplus' exists, if demographic values equal zero for a fraction of the population. Here, removing $x_{2}-x_{1}$ individuals from a population at size $x_{2}$ does not affect the subsequent population size at all. (C) Assuming that the mapping $f$ describes the total dynamics of the population over all seasons, equilibrium population sizes can be determined: here, harvesting the population until the size $x_{1}$ is reached leads to a drop of the equilibrium population size from $x^{*}$ to $x_{2}$. The function $f^{\prime}(x)$ shows the horizontal region of surplus individuals in the map that includes the reduction of $H=x-$ $x_{1}$ individuals when $x$ exceeds $x_{1}$.
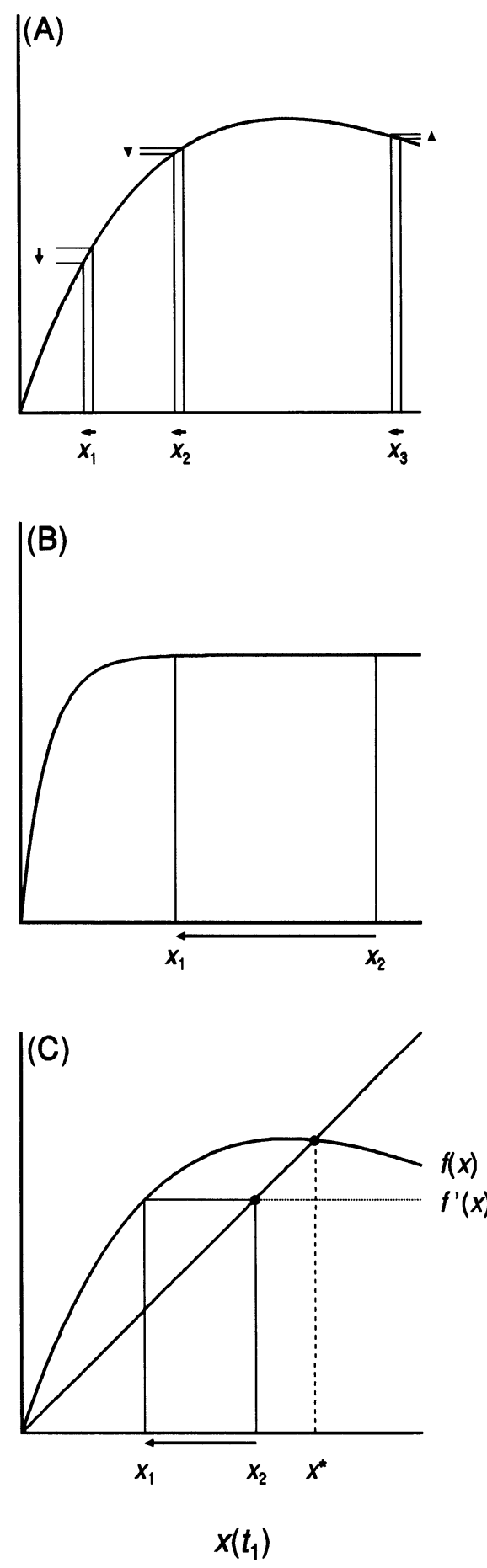

Fig. 3. 
from $x_{2}$ to $x_{1}$ individuals, with a reduction of post-breeding population size equilibrium from $x^{*}$ to $x_{2}$. All culled individuals have positive $v_{D}(x)$ values in the original map, but the population remains in a stable equilibrium from year to year. A modified map, which includes the effect of hunting such that it depicts the removal of any individuals after population size $x_{1}$, will give $v_{D}(x)=0$ for all individuals for which $x \geq x_{1}$ (Fig. $3 \mathrm{C}$, dashed line), so that a surplus with $v_{D}=0$ is established by harvesting itself. However, this comes naturally along with a decrease in the stable population size.

The differential effects of culling individuals within the season in the Ricker and Beverton-Holt models can be explained by an increase in the $v_{D}(x)$ value of a survived individual over time in the mortality season (Fig. 4). The form of $v_{D}(x)$ values give important information. First, the importance of harvest timing is stronger than differentiating between additive (Ricker) and compensatory (Beverton-Holt) mortality. Second, if breeding success is not density-dependent, the value of an individual approaches $\lambda_{0}$ at the start of the breeding season: removing one individual just prior to breeding corresponds to removing all the expected offspring as well. With density dependence acting in both seasons, demographic values never reach as high values. Also, because of the complex form of interacting densities, it is not necessarily true that individuals from a larger population have smaller $v_{D}(x)$ values (Fig. 4D). In every case, however, demographic values increase as the season proceeds, and the demographic value removed, $\Sigma v_{D}(x)$, is minimized if harvesting occurs as early in the season as possible.

Likewise, the analysis can be extended to situations where the mortality rate varies within the season (Fig. 5). The rise in value is steepest during the time when mortality rates reach their peak. Consequently, with such within-season variation, the exact timing of harvesting before the natural peak of mortality matters relatively little compared to the effects of extending the harvest season to include the peak. Both with uniform (Fig. 4) and non-uniform (Fig. 5) mortality, the Beverton-Holt model shows less variation in $v_{D}$ related to the initial population size, which is a sign of compensatory ability. Still, the question of compensation versus addition of mortality seems less important than the progress of mortality during the season, especially so when breeding also obeys some density dependence (small difference between Beverton-Holt and Ricker models, Fig. 4CD).

\section{Discussion}

Any relationship between individual numbers and their survival during the progress of a season (e.g. the over-wintering period) will lead to a relationship between the initial and final population size, such as the examples of deriving the Beverton-Holt and Ricker models (Eqs. (5a) and (5b)) demonstrate. However, the mere relationship between these two time points do not suffice to specify the reaction of the population to added mortality such as harvesting. The resulting population response will depend on the changes of individual survival prospects to removal of other individuals within the season. Such an effect cannot be captured with a single discrete-time equation.

In this study we have demonstrated the importance of the interplay of the structure of densitydependent survival and fecundity and the timing of density-independent actions such as culling a specified harvesting quota. The basic principles are simple. Without the capacity of populations to recover from downward fluctuations, no sustainable harvesting is possible. The successful management of a renewable resource is based on the concept of a sustainable yield, i.e. an exploitation that does not threaten the long-term persistence of the population.

We show the relationship both between harvest timing and the maximum sustainable yield MSY and between harvest timing and population size decrease at yields smaller than the MSY. Regardless of which approach is used, the timing of harvesting is shown to have enough significance that it should not be neglected in population models. The concept of the MSY has been criticized with reason: it represents an unstable equi- 
Beverton-Holt

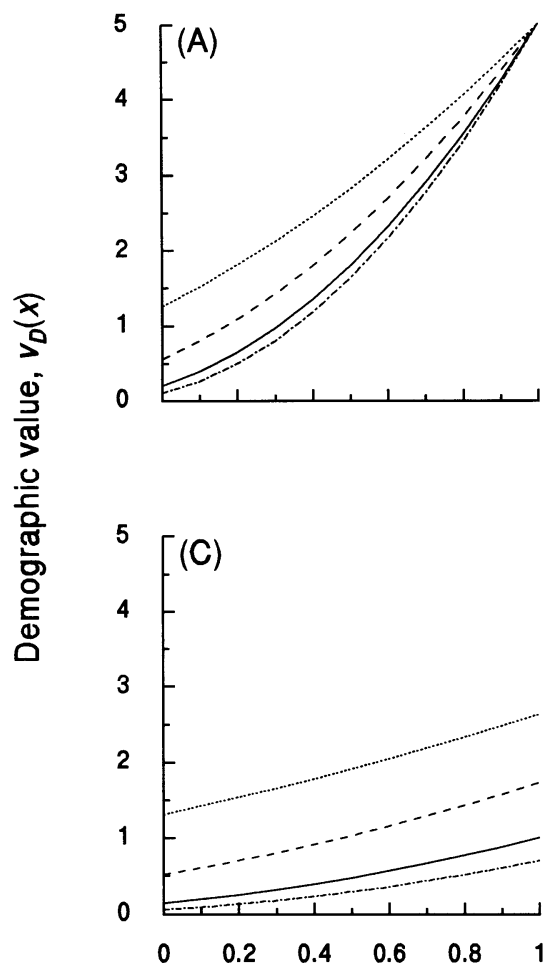

Ricker
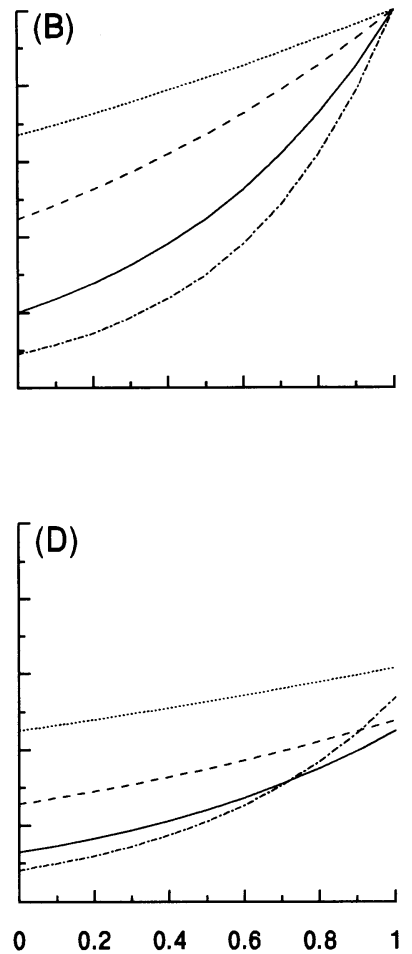

Timing of removal

Fig. 4. Demographic values $v_{D}(x)$ within the mortality season, with the reference time set to the end of the following breeding season. (A) and (C), Beverton-Holt density dependence in the mortality season, (B) and (D), Ricker density dependence in the mortality season; $\lambda_{0}$ and $\mu_{0}$ as in Fig. 1 in both cases. (A) and (B), no density dependence in the breeding season (breeding function $B: x \rightarrow 5 x)$, while in (C) and (D), the per capita reproduction is density-dependent, $B: x \rightarrow 5\left(1-\exp (-0.0001 x)\right.$. Values of $v_{D}(x)$ are given for $x(t)$ that result from $x(0)=0.25 x^{*}$ (dotted line), $0.50 x *$ (dashed line), $1.00 x *$ (solid line), and $1.50 x *$ (dashdot), where $x *$ is the equilibrium population size with no hunting.

librium, making it practically impossible to harvest the population at such a high rate. It should be more correctly interpreted as an absolute upper limit which cannot be exceeded without seriously threatening population persistence (Cook et al., 1997). Our results show that the timing of harvesting has a strong impact both on the MSY and on population responses at lower harvesting pressures.

Our results also emphasize the need to reassess even the more conservatively-derived harvest recommendations, that already take stochasticity and erroneous population size assessment into account
(Ludwig and Walters, 1981; Walters, 1986; Murawski and Idoine, 1989; Thompson, 1992; Ludwig et al., 1993; Frederick and Peterman, 1995; Lande et al., 1995; Sæther et al., 1996), to a lower level if the timing of harvesting is sub-optimal. The assumption that hunting occurs in a single stroke at the end of the breeding season like $H_{a}$ in Eq. (8), will overestimate the sustainable yield no matter what the form of population growth. We especially note the danger of overestimating the tolerable number of kills if the harvesting strategy is based either on too simple (i.e. discrete) population models, or on empirically-based estimates of 
population responses on harvest levels, if the timing of harvesting is subsequently changed.

We propose that a harvesting scheme should consider the demographic values $v_{D}(x)$ of individuals. This concept is related to the reproductive value (Caswell, 1989), as it measures the contribution of an individual to population growth, but specifically focuses on the effects of density dependence. Thus, the demographic value will not remain constant if the individual is one of a population near the carrying capacity, or if it is among the remaining ones when half of the population has already been removed within the hunting season.

The calculation of the demographic value of an individual does not yet provide a direct measure to

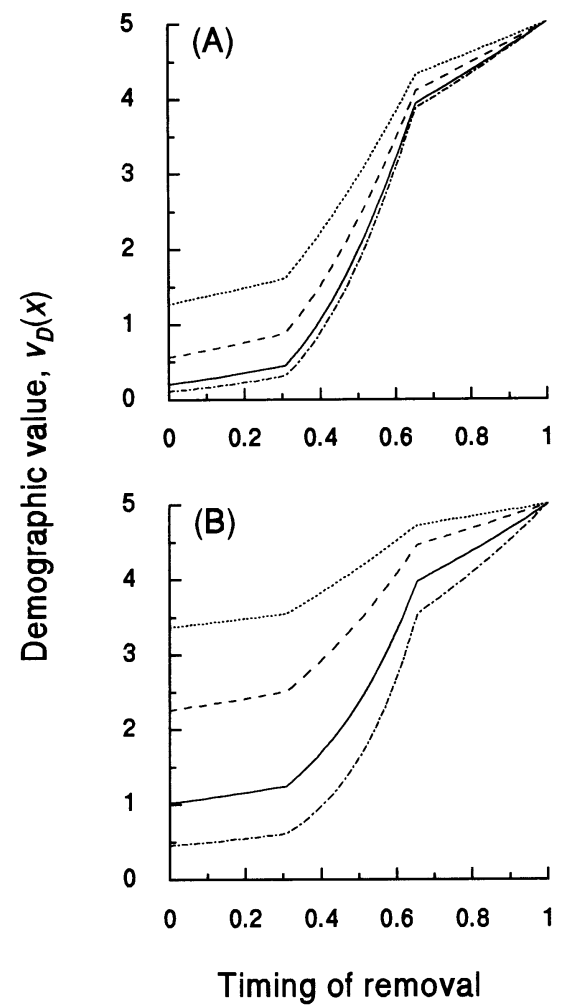

Fig. 5. Demographic values $v_{D}(x)$ within the mortality season for (A) Beverton-Holt and (B) Ricker type density dependence, with an increase in the mortality rate during mid-winter: $\mu_{0}(t)=0.0005$ for $0.33 \leq t \leq 0.66$, and $\mu_{0}(t)=0.0001$ elsewhere. Other parameters are as in Fig. 4. The demographic values are now fairly insensitive to timing before the harshest season begins. Again, because of compensation, the BevertonHolt model shows much less dependence of $v_{D}$ on the initial population size than the Ricker model. assess the sustainability of the chosen harvest. However, in a situation with alternatives available for harvest, there is naturally a trade-off between the yield and the size of the remaining population. We argue that management can be optimized by considering instead the trade-off between the yield and the sum of demographic values, $\Sigma v_{D}(x)$, removed from the population. Not all harvest strategies are Pareto optimal, i.e., fully exploit this trade-off. Therefore, if the form and timing of density dependence is known, diminishing $\Sigma v_{D}(x)$ of culled individuals may be possible without reducing the yield, by seeking Pareto optimal solutions.

Correct harvest timing is one option available to optimizing harvest. However, $v_{D}(x)$ values not only change with time, but are also likely to vary among individuals. Individual contributions to population growth often depend strongly on sex and age as well, and such knowledge can be used to maximize yield while leaving population growth largely unaffected (Caughley, 1977). Such differences among individuals are reflected in their demographic values as well. Specifically, considering a sufficiently long reference time span, demographic values will be proportional to reproductive values in an exponentially growing population where density dependence can be neglected. With density dependence, more complex relationships are possible. As an example, young individuals without a territory may truly represent a 'doomed surplus' at high population densities in territorial species. In any case, specifying the relationship between the planned action of exploitation and the resulting change of population size forms the basis of an optimal harvesting policy. The more accurately the selection of individuals can be determined, and timing of their removal controlled, the higher the sustainable yield that can be maintained in realistic conditions.

\section{Acknowledgements}

We thank Niclas Jonzén and Per Lundberg for discussions. Funding was provided by the Academy of Finland, the Maj and Tor Nessling Foundation, the Emil Aaltonen Foundation, and the Finnish Game Foundation. 


\section{Appendix A}

For the Ricker model, Eq. (4) becomes

$x(T)= \begin{cases}x(0) \mathrm{e}^{-\mu_{0} x(0) T} & 0 \leq T<T_{1} \\ x(0) \mathrm{e}^{-\mu_{0} x(0) T}-\frac{h}{\mu_{0} x(0)}\left(1-\mathrm{e}^{-\mu_{0} x(0)\left(T-T_{1}\right)}\right) & T_{1} \leq T<T_{2} \\ x(0) \mathrm{e}^{-\mu_{0} x(0) T}-\frac{h}{\mu_{0} x(0)}\left(\mathrm{e}^{-\mu_{0} x(0)\left(T-T_{2}\right)}-\mathrm{e}^{-\mu_{0} x(0)\left(T-T_{1}\right)}\right) & T_{2} \leq T<1\end{cases}$

where $h=H /\left(T_{2}-T_{1}\right)$, as above. The equilibrium condition thus becomes

$x(0)=x(1)=\lambda_{0}\left(x(0) \mathrm{e}^{-\mu_{0} x(0)}-\frac{H}{\mu_{0} x(0)\left(T_{2}-T_{1}\right)}\left(\mathrm{e}^{-\mu_{0} x(0)\left(1-T_{2}\right)}-\mathrm{e}^{-\mu_{0} x(0)\left(1-T_{1}\right)}\right)\right)$

The corresponding Beverton-Holt solution (Eq. (3)) is

$x(T)= \begin{cases}\left(\frac{1}{x(0)}+\mu_{0} T\right)^{-1}, & 0 \leq T<T_{1} \\ \sqrt{\frac{h}{\mu_{0}} \tan \left(\arctan \left(\frac{\mu_{0} x(0)}{\sqrt{h \mu_{0}}\left(1+\mu_{0} x(0) T_{1}\right)}\right)-\sqrt{h \mu_{0}}\left(T-T_{1}\right)\right),} & T_{1} \leq T<T_{2} \\ \left(\frac{1}{x\left(T_{2}\right)}+\mu_{0}\left(T-T_{2}\right)\right)^{-1} & T_{2} \leq T<1\end{cases}$

where $x\left(T_{2}\right)=\sqrt{\frac{h}{\mu_{0}}} \tan \left(\arctan \left(\frac{\mu_{0} x(0)}{\sqrt{h \mu_{0}}\left(1+\mu_{0} x(0) T_{1}\right)}\right)-\sqrt{h \mu_{0}}\left(T_{2}-T_{1}\right)\right)$,

such that the equilibrium condition becomes

$x(0)=x(1)=\lambda_{0}$

$\cdot\left(\left(\sqrt{\frac{H}{\mu_{0}\left(T_{2}-T_{1}\right)}} \tan \left(\arctan \left(\frac{\mu_{0} x(0)}{\sqrt{\frac{H \mu_{0}}{\left(T_{2}-T_{1}\right)}\left(1+\mu_{0} x(0) T_{1}\right)}}\right)-\sqrt{\frac{H \mu_{0}}{\left(T_{2}-T_{1}\right)}}\left(T_{2}-T_{1}\right)\right)\right)-1+\mu_{0}\left(1-T_{2}\right)\right)^{-1}$

\section{References}

Allen, L.J.S., Strauss, M.J., Thorvilson, H.G., Lipe, W.N., 1991. A preliminary mathematical model of the apple twig borer (Coleoptera: Bostrichidae) and grapes on the Texas High Plains. Ecol. Model. 58, 369-382.

Anderson, D.R, Burnham, K.P., 1976. Population ecology of the mallard VI, the effect of exploitation on survival. US Fish Wildl. Serv. Resour. Publ. 128, 1-66.

Åström, M., Lundberg, P., Lundberg, S., 1996. Population dynamics with sequential density dependencies. Oikos 75, $174-181$.
Barker, R.J., Hines, J.E., Nichols, J.D., 1991. Effect of hunting on annual survival of grey ducks in New Zealand. J. Wildl. Manage. 55, 260-265.

Beverton, R.J.H., Holt, S.J., 1957. On the dynamics of exploited fish populations. Fish. Inv. II 19, 1-533.

Burnham, K.P., Anderson, D.R., 1984. Tests of compensatory versus additive hypotheses of mortality in mallards. Ecology $65,105-112$.

Burnham, K.P., White, G.C., Anderson, D.R., 1984. Estimating the effect of hunting on annual survival rates of adult mallards. J. Wildl. Manage. 48, 350-361.

Caswell, H., 1989. Matrix Population Models. Sinauer, Sunderland. 
Caughley, G., 1977. Analysis of Vertebrate Populations. Wiley, London.

Clutton-Brock, T.H., Major, M., Guinness, F.E., 1985. Population regulation in male and female red deer. J. Anim. Ecol. 54, 831-846.

Clutton-Brock, T.H., Illius, A.W., Wilson, K., et al., 1997. Stability and instability in ungulate populations: an empirical analysis. Am. Nat. 149, 195-219.

Conroy, M.J., Eberhardt, R.T., 1983. Variation in survival and recovery rates of ring-necked ducks. J. Wildl. Manage. 47, 127-137.

Cook, R.M., Sinclair, A., Stefánsson, G., 1997. Potential collapse of North Sea cod stocks. Nature 385, 521-522.

Doubleday, W.G., 1975. Harvesting in matrix population models. Biometrics 31, 189-200.

Dusek, G.L., Wood, A.K., Stewart, S.T., 1992. Spatial and temporal patterns of mortality among female white-tailed deer. J. Wildl. Manage. 56, 645-650.

Ekman, J., 1984. Density-dependent seasonal mortality and population fluctuations of the temperate zone willow tit (Parus montanus). J. Anim. Ecol. 53, 119-134.

Ellison, L.N., 1991. Shooting and compensatory mortality in tetraonids. Ornis Scand. 22, 229-240.

Errington, P.L., 1934. Vulnerability of Bobwhite populations to predation. Ecology 15, 110-127.

Frederick, S.W., Peterman, R.M., 1995. Choosing fisheries harvest policies: when does uncertainty matter? Can. J. Fish. Aquat. Sci. 52, 291-306.

Getz, W.M., 1980. The ultimate sustainable yield problem in non-linear age-structured populations. Math. Biosci. 48, 279-292.

Getz, W.M., Haight, R.G., 1989. Population Harvesting. Princeton University, Princeton.

Goodyear, C.P., 1996. Variability of fishing mortality by age: consequences for maximum sustainable yield. N. Am. J. Fish. Manage. 16, 8-13.

Hellgren, E.C., Synatzske, D.R., Oldenburg, P.W., Guthery, F.S., 1995. Demography of a collared peccary population in south Texas. J. Wildl. Manage. 59, 153-163.

Hudson, P.J., 1992. Grouse in Space and Time. Game Conservancy, Hampshire, UK.

Kokko, H., Lindström, J., Ranta, E., 1997. Risk analysis of hunting of seal populations in the Baltic. Conserv. Biol. 11, 917-927.

Kot, M., Schaffer, W.M., 1984. The effect of seasonality on discrete models of population growth. Theor. Popul. Biol. 26, 340-360.

Lande, R., Engen, S., Sæther, B.E., 1995. Optimal harvesting of fluctuating populations with a risk of extinction. Am. Nat. 145, 728-745.

Ludwig, D., Hilborn, R., Walters, C.J., 1993. Uncertainty, resource exploitation and conservation: lessons from history. Science $260,17-36$.
Ludwig, D., Walters, C.J., 1981. Measurement errors and uncertainty in parameter estimates for stock and recruitment. Can. J. Fish. Aquat. Sci. 38, 711-729.

MacArthur, R.H., 1960. On the relation between reproductive value and optimal predation. Proc. Natl. Acad. Sci. USA 46, 143-145.

Murawski, S.A., Idoine, J.S., 1989. Yield sustainability under constant-catch policy and stochastic recruitment. Trans. Am. Fish. Soc. 118, 349-367.

Payne, N.F., 1984. Mortality rates of beaver in Newfoundland. J. Wildl. Manage. 48, 117-126.

Reed, W.J., 1980. Optimum age-specific harvesting in a nonlinear population model. Biometrics 36, 579-593.

Rexstad, E.A., 1992. Effect of hunting of annual survival of Canada geese in Utah. J. Wildl. Manage. 56, 297-305.

Ricker, W.E., 1954. Stock and recruitment. J. Fish. Res. Board Can. 11, 559-623.

Robertson, P.A., Rosenberg, A.A., 1988. Harvesting gamebirds. In: Hudson, P.J., Rands, M.R.W. (Eds.), Ecology and Management of Gamebirds. BSP, Oxford, pp. 177201.

Rodriguez, D.J., 1988. Models of growth with density regulation in more than one life stage. Theor. Popul. Biol. 34, 93-113.

Roseberry, J.L., 1979. Bobwhite population responses to exploitation: real and simulated. J. Wildl. Manage. 43, 285305.

Sæther, B.E., Engen, S., Lande, R., 1996. Density-dependence and optimal harvesting of fluctuating populations. Oikos $76,40-46$.

Sedinger, J.S., Rexstad, E.A., 1994. Do restrictive harvest regulations result in higher survival rates in mallards? A comment. J. Wildl. Manage. 58, 571-577.

Skogland, T., 1985. The effects of density-dependent resource limitations on the demography of wild reindeer. J. Anim. Ecol. 54, 359-374.

Small, R.J., Holzwart, J.C., Rusch, D.H., 1991. Predation and hunting mortality of ruffed grouse in central Wisconsin. J. Wildl. Manage. 55, 512-520.

Smith, G.W., Reynolds, R.E., 1992. Hunting and mallard survival, 1979-88. J. Wildl. Manage. 56, 306-316.

Smith, G.W., Reynolds, R.E., 1994. Hunting and mallard survival: a reply. J. Wildl. Manage. 58, 578-581.

Thompson, G.G., 1992. A Bayesian approach to management advice when stock-recruitment parameters are uncertain. Fish. Bull. 90, 561-573.

Walters, C., 1986. Adaptive management of renewable resources. Macmillan, New York.

Yodzis, P., Kolenosky, G.B., 1986. A population dynamics model of black bears in east-central Ontario. J. Wildl. Manage. 50, 602-612. 